\title{
OPEN Molecular characterization and antifungal activity against non-dermatophyte molds causing onychomycosis
}

\author{
Keyvan Pakshir ${ }^{1,2}$, Mandana Kamali ${ }^{1}$, Hasti Nouraei ${ }^{1}$, Kamiar Zomorodian ${ }^{1,2}$, \\ Marjan Motamedi ${ }^{1}$ \& Mozhgan Mahmoodi ${ }^{1}$
}

Onychomycosis is a fungal disease that caused by different types of fungi. Non-dermatophyte molds are a large saprophytic fungi group that live in nature and could affect traumatic nails. The aim of this study was to identify non-dermatophyte molds causing onychomycosis and evaluation of several antifungal activities against the isolates. The samples consisted of 50 non-dermatophyte molds isolated from patients with onychomycosis confirmed by direct and culture examination fungal. DNA was extracted, amplified, and sequenced. Disk diffusion method was used to evaluate itraconazole, fluconazole, ketoconazole, terbinafine, posaconazole, and econazole activity against the isolates. The species identified as: Aspergillus flavus 22 (44\%), A. niger 12 (24\%), A. fumigates, $3(6 \%)$, A. sydowii $3(6 \%)$, A. terreus 1 (2\%), Penicillium commune $2(4 \%)$, P. glabrum $2(4 \%)$, P. chrysogenum, $1(2 \%)$, Fusarium solani $3(6 \%)$ and $F$. thapsinum $1(2 \%)$. Most of the samples were sensitive to terbinafine, itraconazole, and econazole and $94 \%$ of the isolates were resistant to fluconazole. This study showed that Aspergillus species were the most common cause of non-dermatophyte mold onychomycosis and fluconazole was the most resistant antifungals. Care must be taken to choose the appropriate antifungal drug for a better cure.

Fungal infections of nails is called onychomycosis and is one of the most common global nail disorder that may present on toe and fingernails, mostly on toenails ${ }^{1}$. This disorder included up to $18-50 \%$ of all nail diseases and $30 \%$ of cutaneous fungal infections ${ }^{2}$. Clinical signs included changing the color and thickening of the nail, hyperkeratosis, and onycholysis ${ }^{3}$. Although onychomycosis is not life-threatening, this is an important public health concern because of its high prevalence and poor response to therapy ${ }^{4}$. Risk factors for onychomycosis include advancing age, nail trauma or psoriasis, running or swimming, immunosuppression, diabetes, poor peripheral circulation, and fungal infection history elsewhere on the body ${ }^{5,6}$. Among these, trauma caused by outdoor activities in males and hand wet work in females are the major predisposing risk factors for the development of onychomycosis ${ }^{7}$. The tropics area with a wet humid climate that helps fungal growth, sporting, and low awareness of the mycotic disease by the general population may be a possible explanation for the high prevalence rate of onychomycosis ${ }^{7}$.

Onychomycosis is caused by dermatophytes, non dermatophyte (saprophytic) molds (NDMs), or yeasts ${ }^{2}$. The prevalence of NDMs isolated from onychomycosis in various parts of the world ranges between 1.49 and $33.5 \%{ }^{8,9}$. This difference may be due to a difference in the geographical distribution of NDMs, diagnostic criteria, and methods for diagnosis.

The most common NDM agents are Aspergillus spp., Scopulariopsis spp., Alternaria spp., Acremonium spp., and Fusarium spp. that are responsible for approximately $2-25 \%$ of all the agents of onychomycosis ${ }^{8,10}$. Aspergillus spp. are increasingly being reported as primary causative agents of NDMs onychomycosis worldwide, with prevalence as high as 34.4\% in Guatemala ${ }^{11}, 69.3 \%$ in Iran $^{12}$. NDMs agents especially Aspergillus spp. isolated from infected nails are not susceptible to most of the topical and systemic antifungals ${ }^{13}$.

Cure NDMs onychomycosis is difficult, and treatment often is based on oral and topical antifungal therapy while in some cases, topical antifungals may be more effective than oral antifungals, and this may depend on the causative organism ${ }^{14,15}$. Currently, there are five accepted classes of antifungal drugs that are related to

\footnotetext{
${ }^{1}$ Department of Parasitology and Mycology, School of Medicine, Shiraz University of Medical Sciences, Shiraz, Iran. ${ }^{2}$ Basic Sciences in Infectious Diseases Research Center, School of Medicine, Shiraz University of Medical Sciences, Shiraz, Iran. ${ }^{\varpi}$ email: pakshirk@gmail.com
} 


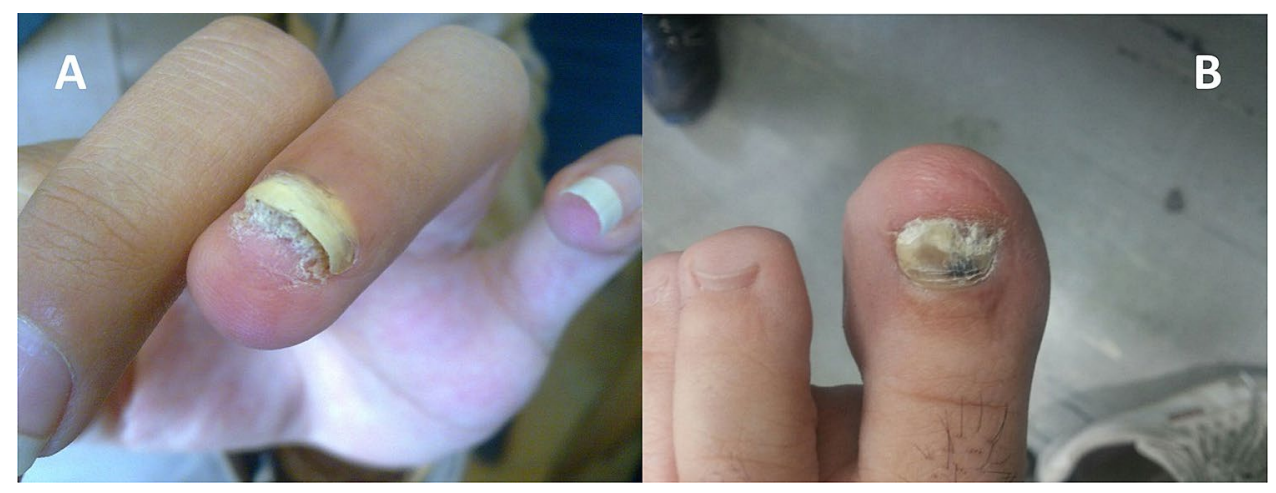

Figure 1. (A) Distal subungual onychomycosis (DSO): whitish discoloration, onycholysis and subungual hyperkeratosis. (B) Total dystrophy and discoloration in toenail.

\begin{tabular}{|l|l|l|l|l|}
\hline \multirow{2}{*}{ Genus (number (\%)) } & \multicolumn{3}{|l|}{ Culture method } & \multicolumn{2}{l|}{ Sequencing method } \\
\cline { 2 - 5 } & Species & Number (\%) & Species & Number (\%) \\
\hline \multirow{4}{*}{$\begin{array}{l}\text { Aspergillus } \\
(41 \text { (82\%)) }\end{array}$} & Flavus & $22(44 \%)$ & Flavus & $22(44 \%)$ \\
\cline { 2 - 5 } & Niger & $12(24 \%)$ & Niger & $12(24 \%)$ \\
\cline { 2 - 5 } & Unknown & $7(14 \%)$ & Fumigatus & $3(6 \%)$ \\
\cline { 2 - 5 } & & & Sydowii & $3(6 \%)$ \\
\cline { 2 - 5 } & & & Terreus & $1(2 \%)$ \\
\hline \multirow{3}{*}{\begin{tabular}{l} 
Penicillium $(10 \%))$ \\
\cline { 2 - 5 }
\end{tabular}} & Unknown & $5(10 \%)$ & Commune & $2(4 \%)$ \\
\cline { 2 - 5 } $\begin{array}{l}\text { Fusarium } \\
(4(8 \%))\end{array}$ & & & Glabrum & $2(4 \%)$ \\
\cline { 2 - 5 } & & & Chrysogenum & $1(2 \%)$ \\
\cline { 2 - 5 } & & & Solani & $3(6 \%)$ \\
\hline
\end{tabular}

Table 1. Known non-dermatophytic molds in cases of onychomycosis in this study based on culture and Sequencing methods.

onychomycosis: allylamines such as terbinafine, azoles such as itraconazole, morpholines, hydroxypyridinones (ciclopirox), and echinocandins ${ }^{16}$. The diagnosis of onychomycosis is conventionally direct microscopy and fungal culture; however, culture-based detection methods may take a few weeks for definite identification. Molecular method techniques using polymerase chain reaction (PCR) assay provide a rapid, stable, and accurate alternative for identifying pathogenic fungi in the affected nail samples of patients with onychomycosis ${ }^{17}$.

Onychomycosis's causative agents may vary depending upon geographic or temporal distribution, and inadequate treatment may lead to infection resistance and recurrence. Proper clinical diagnosis, laboratory workup, and adequate antifungal therapy are thus the standard of care for nails infections, so this study was aimed to find out the pattern of NDMs as causative agents of onychomycosis based on DNA sequence analysis of internal transcribed spacer (ITS) ribosomal DNA (rDNA) and evaluation of antifungal drug activities by disk diffusion methods.

\section{Results}

In this study, the patients included 39 (78\%) females and 11 (22\%) males, and the samples included 39 (78\%) toenails and 11 (22\%) fingernails (Fig. 1).

The isolated agents and their abundance based on conventional methods were classified into three genus; Aspergillus spp. (82\%), Penicillium spp. (10\%) and Fusarium spp. (8\%) (Table 1). Of 50 NDMs isolates, 16 (32\%) cases did not identify at the species level by these methods.

The BLAST analysis of the 50 DNA sequence results indicated that Aspergillus flavus was the most frequently species (44\%), followed by A. niger (24\%), A. fumigatus (6\%), A. sydowii (6\%), A. terreus (2\%), Penicillium commune (4\%), P. glabrum (4\%), P. chrysogenum (2\%), Fusarium solani (6\%) and F. thapsinum (2\%) (Table 1).

The results of culture and sequencing indicate that the three genus of Aspergillus, Penicillium, and Fusarium were identified equally in both methods. Two species of flavus and niger were identified equally at the species level, but the other unknown species could identify by the sequencing method (Fig. 2).

The antifungal drugs patterns (susceptible, intermediate, and resistant) against NDMs is shown in Table 2. The highest sensitivity was observed in terbinafine, itraconazole, and econazole, respectively. Of all these sensitive isolates, two cases were intermediate to terbinafine and econazole, and 1 case was resistant, and 1 case was intermediate to itraconazole. Forty-two isolates (84\%) were sensitive to posaconazole, and the remaining were 


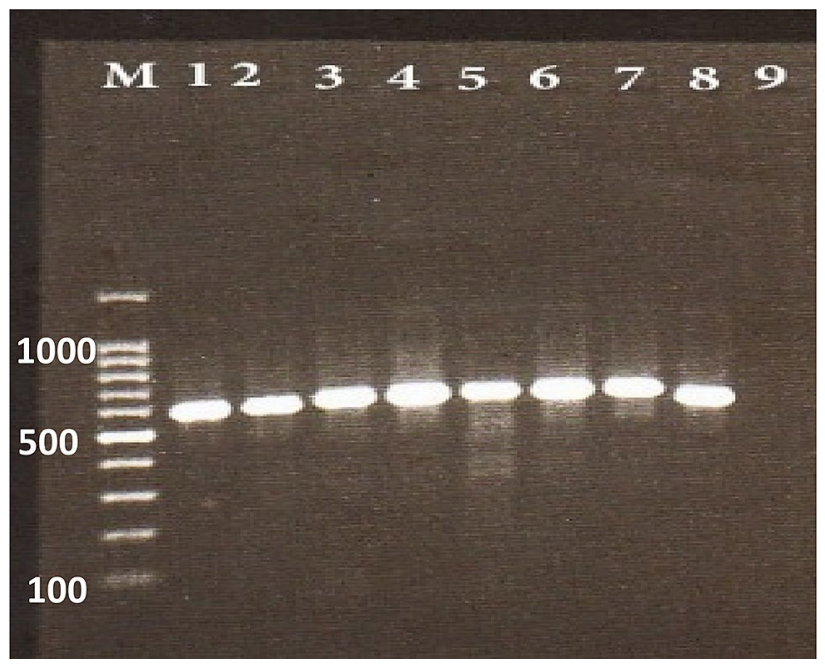

Figure 2. Representative agarose gel electrophoresis of PCR products. Lanes 1, 2: Penicillium spp; Lane 3: Aspergillus sp; Lanes 4: Fusarium sp; Lanes 5-8: Aspergillus spp; lane 9: negative controls and lanes M is the 100bp molecular size marker.

\begin{tabular}{|c|c|c|c|c|c|}
\hline \multirow[b]{2}{*}{ Antifungal drugs } & \multirow[b]{2}{*}{ Number } & \multirow[b]{2}{*}{ Pattern } & \multicolumn{3}{|l|}{ Genus } \\
\hline & & & Aspergillus & Penicillium & Fusarium \\
\hline \multirow{3}{*}{ Posaconazole } & $42(84 \%)$ & S & $36(87.8 \%)$ & $4(80 \%)$ & $2(50 \%)$ \\
\hline & - & I & - & - & - \\
\hline & $8(16 \%)$ & $\mathrm{R}$ & $5(12.2 \%)$ & $1(20 \%)$ & $2(50 \%)$ \\
\hline \multirow{3}{*}{ Itraconazole } & $48(96 \%)$ & $S$ & $40(97.56 \%)$ & $5(100 \%)$ & $3(75 \%)$ \\
\hline & $1(2 \%)$ & I & - & - & $1(25 \%)$ \\
\hline & $1(2 \%)$ & $\mathrm{R}$ & $1(2.44 \%)$ & - & - \\
\hline \multirow{3}{*}{ Fluconazole } & $1(2 \%)$ & $S$ & - & - & $1(25 \%)$ \\
\hline & $2(4 \%)$ & I & - & $2(40 \%)$ & - \\
\hline & $47(94 \%)$ & $\mathrm{R}$ & $41(100 \%)$ & $3(60 \%)$ & $3(75 \%)$ \\
\hline \multirow{3}{*}{ Terbinafine } & $48(96 \%)$ & $S$ & $41(100 \%)$ & $5(100 \%)$ & $2(50 \%)$ \\
\hline & $2(4 \%)$ & I & - & - & $2(50 \%)$ \\
\hline & - & $\mathrm{R}$ & - & - & - \\
\hline \multirow{3}{*}{ Ketoconazole } & $36(72 \%)$ & $S$ & $30(73.8 \%)$ & $5(100 \%)$ & $2(50 \%)$ \\
\hline & $13(26 \%)$ & I & $11(26.82 \%)$ & - & $2(50 \%)$ \\
\hline & $1(2 \%)$ & $\mathrm{R}$ & - & - & - \\
\hline \multirow{3}{*}{ Econazole } & $48(96 \%)$ & $S$ & $39(95.13 \%)$ & $5(100 \%)$ & $3(75 \%)$ \\
\hline & $2(4 \%)$ & I & $2(4.87 \%)$ & - & $1(25 \%)$ \\
\hline & - & $\mathrm{R}$ & - & - & - \\
\hline
\end{tabular}

Table 2. Antifungal susceptibility pattern of non-dermatophytic molds disk diffusion method. S: susceptible, I: intermediate, R: resistant.

resistant (16\%). The results showed that 36 isolates (72\%) were sensitive to ketoconazole, 13 isolates $(26 \%)$ were intermediate, and one isolate (2\%) was resistant (Fig. 3).

Based on the criteria of sensitivity and resistance, the highest resistance was observed in fluconazole, and it was statistically significant among other drugs $(p$-value $<0.001)$.

It is noteworthy that 47 out of 50 cases (94\%) were resistant to fluconazole, and only 1 case was sensitive to this drug, and the remaining two were intermediate.

In three genus, 1 Aspergillus, 2 Penicillium, and 2 Fusarium isolates were resistant to posaconazole and fluconazole. There was only one isolate resistant to itraconazole, which was related to A. flavus species (Table 2).

Also, the resistance samples to posaconazole compared to itraconazole and ketoconazole were significant. The $p$-value in both was 0.036 , so the resistance of the samples to posaconazole was higher than itraconazole and ketoconazole. 


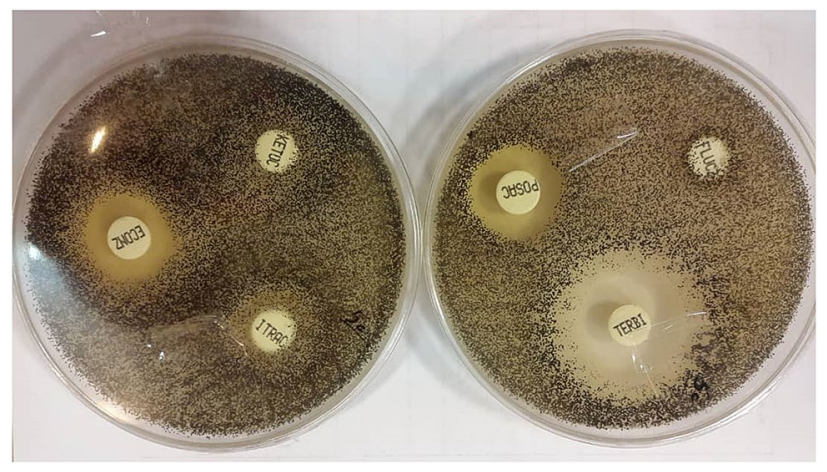

Figure 3. Antifungal sensitivity of Asperigillus niger showing high sensitivity to terbinafine and resistance to fluconazole, ketoconazole and itraconazole.

The most effective drugs were terbinafine, itraconazole, and econazole that $96 \%$ of the samples were sensitive. The next most effective drugs were ketoconazole and posaconazole, which $84 \%$ and $72 \%$ of the samples were sensitive to them, respectively. Sensitive samples to econazole, itraconazole, and terbinafine compared to ketoconazole were statistically significant with a $p$-value of 0.001 , so samples' sensitivity to econazole, itraconazole, and terbinafine were higher than ketoconazole. There was no significant difference in the samples' sensitivity to ketoconazole, econazole, itraconazole, and terbinafine compared to posaconazole ( $p$-value $>0.05)$. The susceptibility of most frequent species (A. flavus and A. niger) to all drugs except ketoconazole and fluconazole was the same ( $p$-value $>0.05$ ), but ketoconazole was found to be more effective in flavus species than niger species $(p$-value $<0.001)$. Both species were determined to have a fluconazole-resistant ratio.

\section{Discussion}

Onychomycosis is one of the most common nail infections in which dermatophytes, yeasts, and non dermatophyte molds have been incriminated as etiological agents. According to the latest reports, the global prevalence of onychomycosis is nearly $2-9 \%^{7}$. Onychomycosis often was mimicked with psoriasis of the nail, eczema, bacterial infections, and contact dermatitis ${ }^{18}$. So, correctly determining the etiologic agents of onychomycosis is important to provide a baseline for administering appropriate antifungal therapy and identifying the source of infection, hence facilitating prevention measures ${ }^{19}$.

At the moment, traditional mycology remains the gold standard for diagnosing NDMs onychomycosis. This includes obtaining positive results from $\mathrm{KOH}$ and culture. The culture method, although it demonstrates the viability and allows the identification of isolated fungi but it is time-consuming, and due to the diversities and similarities among different species, morphological methods are not specific enough. It appears that a more accurate method is critical to differentiate various members of the genus ${ }^{20}$.

In addition to standard procedures, the use of molecular biological techniques such as polymerase chain reaction (PCR), followed by sequencing of appropriate targets, has important implications for understanding these pathogenic fungi ${ }^{21}$. Scopulariopsis brevicaulis, Fusarium species, Aspergillus species, Neoscytalidium dimidiatum, and Acremonium species are the most common causative agents of onychomycosis in the world that we could not isolate some of them such as S. brevicaulis, N. dimidiatum or Acremonium spp. ${ }^{13}$. Comparison of culture and molecular methods indicated that Aspergillus, Penicillium, and Fusarium genus were identified in both methods. Using molecular methods increased the sensitivity and specificity of identification of microorganisms that caused onychomycosis as our 16 unknown isolates were identified by this method as followed: $A$. fumigatus $(\mathrm{n}=3), A$. sydowii $(\mathrm{n}=3)$, A. terreus $(\mathrm{n}=1)$, Penicillium commune $(\mathrm{n}=2)$, P. glabrum $(\mathrm{n}=2)$, P. chrysogenum $(\mathrm{n}=1)$, Fusarium solani $(\mathrm{n}=3)$ and $F$. thapsinum $(\mathrm{n}=1)$.

In this study, A. flavus was responsible for approximately $44 \%$ of all the samples and that this data was agree with Nouripour-Sisakht et al. ${ }^{22}$, Chadeganipour et al. ${ }^{23}$ and Halvaee et al. ${ }^{24}$, they reported that $77.3 \%, 66 \%$ and $60.9 \%$ of their isolates were A. flavus, respectively. The high frequency of nail infections due to $A$. flavus can be attributed to the fact that dry climate in Iran that favors the growth of thermo-tolerant fungi like A. flavus and this species is one of the most frequent Aspergillus species isolated from the environment ${ }^{25,26}$. This genus is one of the common pathogenic fungi in humans ${ }^{27}$. Also, in Hashemi et al. study, based on conventional method, A. flavus reported as predominant agent responsible for NDMs onychomycosis infections ${ }^{28}$. Our study was in contrast with Martinez et al. in Brazil ${ }^{11}$ and Bitew et al. in Ethiopia ${ }^{7}$ that reported A. niger and Neoscytalidium dimidiatum, respectively, as more prevalent species. Differences in patient characteristics, sample size, and study design could be reasons for such discrepancy.

Penicillium commune and P. glabrum that identified were reported for the first time from onychomycosis patients in Iran but also those fungi have been isolated from environmental area of Mazandaran province in north of Iran previously and, identified by beta-tubulin gene sequencing method ${ }^{29}$. It is hard to identify these organism by conventional methods maybe for this reason there was less reports regarding these isolates from onychomycosis around the world. Penicillium chrysogenum as pathogenic fungi is involved in developing skin diseases such as onychomycosis and invasive diseases ${ }^{30}$.

Treatment is necessary for onychomycosis to avoid serious consequences such as secondary bacterial infections, complete nail dystrophy, and cosmetic reasons ${ }^{31}$. It is including use topical and oral agents and physical 
treatments ${ }^{32}$. Drug choice depends on several things such as the severity of nail damage, type of organism, adverse effects, and success or failure of previous treatments ${ }^{33}$.

Treatment in NDMs onychomycosis is dependent on fungal species that caused infection and their susceptibility to antifungals prescription because different fungi have different responses to drugs. On the other hand, many of the selected therapies were failed because of increasing the prevalence of drug resistance related to the fungi genetic factors and the type of antifungals or host conditions $\mathrm{s}^{33}$.

Drug susceptibility tests have several benefits, such as preventing long-term, unnecessary, and even high-risk drug use and reducing treatment costs ${ }^{34}$.

The disk diffusion method is a simple, inexpensive, and rapid method that qualitatively demonstrates the antifungal drugs' effectiveness ${ }^{35}$. In our study, terbinafine, econazole, and itraconazole were identified as the most effective antifungal drugs against onychomycosis caused by NDMs agents. In fact, out of the 50 isolates, $96 \%$ of them were sensitive to these three antifungals.

Except for several cases of Fusarium solani and Aspergillus flavus all the isolates were sensitive to terbinafine, econazole and itraconazole.

As shown in our in vitro study, terbinafine and itraconazole were the best candidates for NDM onychomycosis treatment, but it needs more details about in vivo data analysis. One reason for the good effects of terbinafine is its appropriate penetration into the nail matrix ${ }^{36}$.

As we showed in this study, all Aspergillus species were sensitive to terbinafine, and this result agreed with Gupta et al. ${ }^{13}$ and Trovato et al. ${ }^{37}$ in Italy and Haghani et al. ${ }^{38}$ and Xu et al. ${ }^{25}$ in Iran. In other study in Iran reported that some Aspergillus species were resistance to terbinafine and this result was not agree with our study ${ }^{39}$.

A noteworthy point in this study was the high resistance of isolates to fluconazole (94\%). All species of Aspergillus were resistant to fluconazole. Our results agreed with Tsang et al ${ }^{40}$ study and were in contrast with Haghani et al. ${ }^{38}$ that reported Aspergillus terreus was sensitive to fluconazole. So, fluconazole is not suitable to cure onychomycosis causing by NDMs but is commonly prescribed in combination with the other antifungals such as itraconazole and terbinafine cases of moderate to severe nail involvement. The results in Falahati et al. study showed that the clinical isolate of $A$. clavatus resistant to the wide spectrum of antifungal drugs such as terbinafine, posaconazole, voriconazole, etc. but sensitive to itraconazole ${ }^{39}$. Due to the side effects and high resistance of NDMs to fluconazole, drug susceptibility testing is important ${ }^{31}$.

It is necessary to mention that two isolates of Fusarium solani were resistant to all five antifungal drugs, except each one of them was sensitive to econazole and itraconazole separately. Bueno et al. also reported Fusarium as resistant fungi to antifungal drugs in his study ${ }^{41}$. Although Fusarium had less frequency than the other genus in our study, special attention should be given to these genus because of their antifungal resistance and keratinolytic activity that helped Fusarium to use nails as a source of nutrition ${ }^{42}$.

The high prevalence rate of onychomycosis with different fungi groups involving etiological agents suggests that more studies should be done on the prevalence. This is for better understanding these issues and could cause developing better preventive procedures and reducing treatment costs. Also, in vivo studies are needed to confirm the effects of drugs in order to find a suitable drug to treat these cases of onychomycosis.

\section{Conclusion}

Onychomycosis is a common multifactorial disease of the nails. The prevalence of fungal nail infections is affected by age, lifestyle, and some underlying diseases. A global outlook on the treatment of onychomycosis provides a framework of success for the clinician. Healthcare could be more effective if physicians prescribe the most effective antifungals for the treatment of onychomycosis.

\section{Materials and methods}

Collection of nail samples. Fifty NDMs onychomycosis cases among 300 clinically suspected patients with onychomycosis referred to medical mycology laboratory enrolled in this study. Patients who had a previous history of antifungal therapy were excluded from the study. Small pieces of the nail debris were collected from the affected area using sterile nail clippers and scalpel.

Mycological examination. A part of nail scrapings was immersed in a drop of $20 \% \mathrm{KOH}$ over a slide and kept in a wet chamber at room temperature for direct microscopy examination.

The other parts of the nail samples were simultaneously inoculated on Sabouraud's dextrose agar (SDA) (Merck, Darmstadt, Germany), SDA containing chloramphenicol $(0.05 \mathrm{mg} / \mathrm{l})$, with and without actidione $(500 \mathrm{mg} / \mathrm{l})$. The cultures were incubated at $28^{\circ} \mathrm{C}$ for $1-2$ weeks. The plates were examined twice weekly for any growth. No growth at the second week was considered as negative. A broad septate/non septate hyphae detection in the direct exam along with positive culture (saprophyte colony growth) was considered as NMD onychomycosis. Genus and species of fungi were identified by colony morphology and microscopic examination by using teas mounts and slide culture. Diagnosis of NDM onychomycosis was made on the basis of the following criteria: (1) nail abnormalities; (2) positive $\mathrm{KOH}$, (3) isolation in culture, (4) repeated isolation in culture (5) failure to isolate a dermatophyte in culture; and in suspicious cases we checked growth of more than 5 colonies of the same mold in at least 2 repeated nail samplings ${ }^{43,44}$.

Preparation of genomic DNA. Total cellular DNA was extracted from a small amount of mycelium cultured on Sabouraud agar slants, by the rapid mini-preparation method ${ }^{45}$. Briefly, to a $1.5 \mathrm{ml}$ eppendorf tube containing $500 \mu \mathrm{l}$ of lysis buffer ( $400 \mathrm{mM}$ Tris- $\mathrm{HCl}[\mathrm{pH}=8], 60 \mathrm{mM}$ EDTA pH $[\mathrm{pH}=8], 150 \mathrm{mM} \mathrm{NaCl}, 1 \%$ [w:v] SDS), a small portion of mycelium grown on SDA in primary culture was added using a sterile toothpick, with which the mycelia were disrupted. The tube was then left at room temperature for $10 \mathrm{~min}$. After adding $150 \mu \mathrm{l}$ of 


\begin{tabular}{|l|l|l|l|l|}
\hline \multirow{2}{*}{ Antifungal tablets } & \multirow{2}{*}{ Potency $(\boldsymbol{\mu g})$} & \multicolumn{3}{|l|}{ Zone diameter in mm } \\
\cline { 3 - 6 } & Sensitive & Intermediate & Resistant \\
\hline Ketoconazol & 15 & $\geq 28$ & $27-21$ & $\leq 20$ \\
\hline Fluconazole & 25 & $\geq 19$ & $15-18(\mathrm{DD})$ & $\leq 14$ \\
\hline Itraconazole & 10 & $\geq 23$ & $14-22(\mathrm{DD})$ & $\leq 13$ \\
\hline Posaconazole & 5 & $\geq 17$ & $14-16(\mathrm{DD})$ & $\leq 13$ \\
\hline Econazole & 1 & $\geq 20$ & $12-19$ & $\leq 11$ \\
\hline Terbinafine & 1 & $\geq 20$ & $12-19$ & $\leq 11$ \\
\hline
\end{tabular}

Table 3. Inhibition zones diameters $(\mathrm{mm})$ of antifungal tablets examined in this study after inoculation of non-dermatophytic mold suspension. $\mathrm{DD}=$ doses dependent.

potassium acetate buffer $\mathrm{pH}[\mathrm{pH}=4.8]$ (5 $\mathrm{M}$ potassium acetate, acetic acid), the tube was vortexed briefly, and cellular debris and precipitated proteins were removed by centrifugation at $>10,000 \mathrm{~g}$ for $1 \mathrm{~min}$. The supernatant was transferred to another $1.5 \mathrm{ml}$ eppendorf tube and centrifuged again as above. After the supernatant was transferred to a new $1.5 \mathrm{ml}$ eppendorf tube, an equal volume of isopropyl alcohol was added. The tube was mixed briefly by inversion, centrifuged at $>10,000 \mathrm{~g}$ for $2 \mathrm{~min}$, and the supernatant was discarded. The resultant DNA pellet was washed in $300 \mathrm{ml}$ of $70 \%$ (v:v) ethanol. After centrifuging at 10,000 $\mathrm{g}$ for $1 \mathrm{~min}$, the supernatant was discarded. The DNA pellet is air-dried and dissolved in $50 \mu \mathrm{l}$ of DW.

Polymerase chain reaction (PCR) amplification. A $6 \mu$ laliquot of template DNA, $0.5 \mu \mathrm{M}$ of each forward (ITS1: 5'-TTC GTA GGT GAA CCT GCG G-3') and reverse primer (ITS4: 5'-TCC TCC GCT TAT TGA TAT GC-3'), $25 \mu$ of premix (Ampliqon, Denmark) and final volume of $50 \mu$ l were used for PCR, under the following conditions: initial denaturation for $5 \mathrm{~min}$ at $94{ }^{\circ} \mathrm{C}$; 32 cycles for denaturation $\left(30 \mathrm{~s}\right.$ at $\left.94{ }^{\circ} \mathrm{C}\right)$; annealing for $45 \mathrm{~s}$ at $56^{\circ} \mathrm{C}$; and extension for $45 \mathrm{~s}$ at $72{ }^{\circ} \mathrm{C}$, followed by an ultimate extension step at $72{ }^{\circ} \mathrm{C}$ for $7 \mathrm{~min}$. The PCR products were electrophoresed on a $1.2 \%$ agarose gel.

Sequencing and sequence analysis. All the amplified PCR products were sequenced unilaterally, using the forward primer (ITS1) as for the primary PCR, via an automated DNA sequencer (Bioneer Company, South Korea). The sequences were edited with Geneious software (www.geneious.com), and for final identification, the obtained consensus sequences were compared with the pubmed database (https://www.ncbi.nlm.nih.gov/ pubmed/).

Antifungal susceptibility. Antifungal susceptibility test by disk diffusion method was done according to CLSI M 51-A document. In brief, the isolates were subcultured on potato dextrose agar (PDA) at $25^{\circ} \mathrm{C}$ for 2-7 days before testing. The conidial inocula were prepared, and the turbidity was adjusted by spectrophotometry to an optical density of 0.09 to 0.13 . Then entire surface of mueller hinton agar (MHA) was inoculated with a sterile cotton swab with the undiluted mold stock inoculum suspension. Antifungal disks (Rosco Diagnostica, Denmark) including ketoconazol $(15 \mu \mathrm{g})$, fluconazole $(25 \mu \mathrm{g})$, itraconazole $(10 \mu \mathrm{g})$, posaconazole $(5 \mu \mathrm{g})$, terbinafine $(1 \mu \mathrm{g})$ and econazole $(1 \mu \mathrm{g})$ were applied to the inoculated media. The plates were incubated at $35^{\circ} \mathrm{C}$ and read after 2-3 days. Inhibition zone diameter were interpreted in accordance with CLSI M51-A (Table 3).

Statistical analysis. Results were analyzed using the SPSS22 (Statistical Package for the Social Sciences) program. Fisher exact test and chi-square were considered statistically significant with a $p$-value less than 0.05 .

Ethical statement. This project was found to be according to the ethical principles and the national norms and standards for conducting Medical Research in Iran and has been approved by the research ethics committee (Shiraz University of Medical Sciences. IR.SUMS.REC.1396.5775).

Informed consent. Informed consent to participate and publish was obtained from all the participants.

\section{Repositories}

The data used to support the findings of this study were supplied by the Vice-Chancellor for Research of Shiraz University of Medical Sciences under license. Requests for data access should be made to Keyvan Pakshir, pakshirk@gmail.com.

Received: 30 August 2021; Accepted: 30 September 2021

Published online: 20 October 2021

\section{References}

1. Solís-Arias, M. P. \& García-Romero, M. T. Onychomycosis in children. A review. Int. J. Dermatol. 56, 123-130 (2017).

2. Adekhandi, S. et al. Incidence and epidemiology of onychomycosis in patients visiting a tertiary care hospital in India. Cutis $\mathbf{9 5}$, E20-25 (2015). 
3. Elewski, B. E. et al. Onchomycosis: An overview. J. Drugs Dermatol. 12, s96-s103 (2013).

4. Christenson, J. K. et al. Challenges and opportunities in the management of onychomycosis. J. Fungi 4, 87 (2018).

5. Lipner, S. R. \& Scher, R. K. Onychomycosis: Clinical overview and diagnosis. J. Am. Acad. Dermatol. 80, 835-851 (2019).

6. Elewski, B. E. \& Tosti, A. Risk factors and comorbidities for onychomycosis: Implications for treatment with topical therapy. J. Clin. Aesthet. Dermatol. 8, 38 (2015).

7. Bitew, A. \& Wolde, S. Prevalence, risk factors, and spectrum of fungi in patients with onychomycosis in Addis Ababa, Ethiopia: A prospective study. J. Trop. Med. 2019 (2019).

8. Moreno, G. \& Arenas, R. Other fungi causing onychomycosis. Clin. Dermatol. 28, 160-163 (2010).

9. Bonifaz, A., Cruz-Aguilar, P. \& Ponce, R. M. Onychomycosis by molds. Report of 78 cases. Eur. J. Dermatol. 17, 70-72 (2007).

10. Bongomin, F., Batac, C., Richardson, M. D. \& Denning, D. W. A review of onychomycosis due to Aspergillus species. Mycopathologia 183, 485-493 (2018)

11. Martínez-Herrera, E. O., Arroyo-Camarena, S., Tejada-García, D. L., Porras-López, C. F. \& Arenas, R. Onychomycosis due to opportunistic molds. An. Bras. Dermatol. 90, 334-337 (2015).

12. Motamedi, M. et al. Growing incidence of non-dermatophyte onychomycosis in Tehran, Iran. Jundishapur J. Microbiol. 9 (2016).

13. Gupta, A. K. et al. Systematic review of nondermatophyte mold onychomycosis: Diagnosis, clinical types, epidemiology, and treatment. J. Am. Acad. Dermatol. 66, 494-502 (2012).

14. Shemer, A. Update: Medical treatment of onychomycosis. Dermatol. Ther. 25, 582-593 (2012).

15. Shemer, A. et al. Continuous terbinafine and pulse itraconazole for the treatment of non-dermatophyte mold toenail onychomycosis. J. Dermatol. Treat., 1-4 (2019).

16. Gupta, A. K. \& Simpson, F. C. Investigational drugs for onychomycosis. Exp. Opin. Investig. Drugs 23, 97-106 (2014).

17. Ebihara, M., Makimura, K., Sato, K., Abe, S. \& Tsuboi, R. Molecular detection of dermatophytes and nondermatophytes in onychomycosis by nested polymerase chain reaction based on $28 \mathrm{~S}$ ribosomal RNA gene sequences. Br. J. Dermatol. 161, 1038-1044 (2009).

18. Holzberg, M. Common nail disorders. Dermatol. Clin. 24, 349-354 (2006).

19. Vasconcellos, C. et al. Identification of fungi species in the onychomycosis of institutionalized elderly. An. Bras. Dermatol. 88, 377-380 (2013).

20. Monod, M. \& Méhul, B. Recent findings in onychomycosis and their application for appropriate treatment. J. Fungi 5, 20 (2019).

21. Joyce, A., Gupta, A. K., Koenig, L., Wolcott, R. \& Carviel, J. Fungal diversity and onychomycosis: an analysis of 8,816 toenail samples using quantitative PCR and next-generation sequencing. J. Am. Podiatr. Med. Assoc. 109, 57-63 (2019).

22. Nouripour-Sisakht, S. et al. Aspergillus species as emerging causative agents of onychomycosis. J. Mycol. Med. 25, 101-107 (2015).

23. Chadeganipour, M., Nilipour, S. \& Ahmadi, G. Study of onychomycosis in Isfahan Iran. Mycoses 53, 153-157 (2010).

24. Halvaee, S. et al. A mycological and molecular epidemiologic study on onychomycosis and determination in vitro susceptibilities of isolated fungal strains to conventional and new antifungals. Front. Cell. Infect. Microbiol. 652 (2021).

25. $\mathrm{Xu}, \mathrm{X}$. et al. Identification and in vitro antifungal susceptibility of causative agents of onychomycosis due to Aspergillus species in Mashhad Iran. Sci. Rep. 11, 1-8 (2021).

26. Diba, K., Mirhendi, H., Kordbacheh, P. \& Rezaie, S. Development of RFLP-PCR method for the identification of medically important Aspergillus species using single restriction enzyme MwoI. Braz. J. Microbiol. 45, 503-507 (2014).

27. Hedayati, M., Pasqualotto, A., Warn, P., Bowyer, P. \& Denning, D. Aspergillus flavus: Human pathogen, allergen and mycotoxin producer. Microbiology 153, 1677-1692 (2007).

28. Hashemi, S. et al. Onychomycosis in Tehran: Mycological study of 504 patients. Mycoses 53, 251-255 (2010).

29. Abastabar, M. et al. Genetic and morphological diversity of the genus penicillium from mazandaran and Tehran provinces, Iran. Jundishapur J. Microbiol. 9 (2016).

30. Guevara-Suarez, M. et al. Penicillium-like fungi from clinical samples in the USA and their antifungal susceptibility. J. Clin. Microbiol. JCM. 00960-00916 (2016).

31. Gupta, A. K., Versteeg, S. G. \& Shear, N. H. Onychomycosis in the 21 st century: An update on diagnosis, epidemiology, and treatment. J. Cutan. Med. Surg. 21, 525-539 (2017).

32. Westerberg, D. P. \& Voyack, M. J. Onychomycosis: Current trends in diagnosis and treatment. Am. Fam. Phys. 88, 762-770 (2013).

33. Thomas, J., Peterson, G. M., Christenson, J. K., Kosari, S. \& Baby, K. E. Antifungal drug use for onychomycosis. Am. J.Ther. 26, e388-e396 (2019).

34. Reiss, E., Shadomy, H. J. \& Lyon, G. M. Fundamental Medical Mycology (Wiley, 2011).

35. Pai, V., Ganavalli, A. \& Kikkeri, N. N. Antifungal resistance in dermatology. Indian J. Dermatol. 63, 361 (2018).

36. Tchernev, G. et al. Onychomycosis: Modern diagnostic and treatment approaches. Wien. Med. Wochenschr. 163, 1-12 (2013).

37. Trovato, L., Rapisarda, M., Greco, A., Galata, F. \& Oliveri, S. In vitro susceptibility of nondermatophyte molds isolated from onycomycosis to antifungal drugs. J. Chemother. 21, 403-407 (2009).

38. Haghani, I., Shams-Ghahfarokhi, M., Dalimi Asl, A., Shokohi, T. \& Hedayati, M. T. Molecular identification and antifungal susceptibility of clinical fungal isolates from onychomycosis (uncommon and emerging species). Mycoses 62, 128-143 (2019).

39. Falahati, M. et al. The first case of total dystrophic onychomycosis caused by Aspergillus clavatus resistant to antifungal drugs. Mycopathologia 181, 273-277 (2016).

40. Tsang, C.-C. et al. Genetic diversity of Aspergillus species isolated from onychomycosis and Aspergillus hongkongensis sp. nov., with implications to antifungal susceptibility testing. Diagn. Microbiol. Infect. Dis. 84, 125-134 (2016).

41. Bueno, J. et al. In vitro activity of fluconazole, itraconazole, voriconazole and terbinafine against fungi causing onychomycosis. Clin. Exp. Dermatol. Exp. Dermatol. 35, 658-663 (2010).

42. Gupta, C. et al. Genotyping and in vitro antifungal susceptibility testing of Fusarium isolates from onychomycosis in India. Mycopathologia 181, 497-504 (2016).

43. Tosti, A., Piraccini, B. M. \& Lorenzi, S. Onychomycosis caused by nondermatophytic molds: Clinical features and response to treatment of 59 cases. J. Am. Acad. Dermatol. 42, 217-224 (2000).

44. Noguchi, H. et al. Non-dermatophyte mould onychomycosis in Japan. Med. Mycol. J. 61, 23-31 (2020).

45. Liu, D., Coloe, S., Baird, R. \& Pedersen, J. Rapid mini-preparation of fungal DNA for PCR. J. Clin. Microbiol. 38, 471-471 (2000).

\section{Author contributions}

Conceptualization: K.P., K.Z.Data curation: M.K.Formal analysis: M.M.Funding acquisition: K.P.Investigation: M.K., H.N.Methodology: M.K.Validation: M.M., H.N., Mo.M.Supervision: K.P., M.M.Writing original article: K.P., H.N., Mo.M.Writing-review and editing: All co-authors.

\section{Competing interests}

The authors declare no competing interests.

\section{Additional information}

Correspondence and requests for materials should be addressed to K.P. 
Reprints and permissions information is available at www.nature.com/reprints.

Publisher's note Springer Nature remains neutral with regard to jurisdictional claims in published maps and institutional affiliations.

(c) (i) Open Access This article is licensed under a Creative Commons Attribution 4.0 International License, which permits use, sharing, adaptation, distribution and reproduction in any medium or format, as long as you give appropriate credit to the original author(s) and the source, provide a link to the Creative Commons licence, and indicate if changes were made. The images or other third party material in this article are included in the article's Creative Commons licence, unless indicated otherwise in a credit line to the material. If material is not included in the article's Creative Commons licence and your intended use is not permitted by statutory regulation or exceeds the permitted use, you will need to obtain permission directly from the copyright holder. To view a copy of this licence, visit http://creativecommons.org/licenses/by/4.0/.

(C) The Author(s) 2021 